\title{
Clinical evidence in the treatment of rotator cuff tears with hyaluronic acid
}

\author{
Leonardo Osti ${ }^{1}$ \\ Matteo Buda² \\ Angelo Del Buono ${ }^{3}$ \\ Raffaella Osti² \\ Leo Massari² \\ 1 Unit of Arthroscopy and Sports Medicine, Hesperia \\ Hospital, Modena, Italy \\ 2 Department of Orthopaedic and Traumatology, Uni- \\ versity of Ferrara, Italy \\ ${ }^{3}$ Department of Orthopaedic and Trauma Surgery \\ Hospital Sant'Anna, Como, Italy
}

Corresponding author:

Leonardo Osti

Unit of Arthroscopy and Sports Medicine, Hesperia Hospital

Via Arquà, $80 / a$

41125 Modena, Italy

E-mail: leonardoosti@yahoo.com

\section{Summary}

Purpose: the aim of this quantitative review is to document potential benefit and adverse effects of hyaluronic acid (HA) injection into the shoulder with rotator cuff tears.

Methods: a systematic literature search was performed in english PubMed, Medline, Ovid, Google Scholar and Embase databases using the combined key words "hyaluronic acid", "rotator cuff tear", "hyaluronate", "shoulder", "viscosupplementation", with no limit regarding the year of publication. Articles were included if they reported data on clinical and functional outcomes, complications in series of patients who had undergone HA injection for management of rotator cuff tears. Two Authors screened the selected articles for title, abstract and full text in accordance with predefined inclusion and exclusion criteria. The papers were accurately analyzed focusing on objective rating scores reported.

Results: a total of 11 studies, prospective, 7 were randomized were included by full text. A total of 1102 patients were evaluated clinically after different HA injection compare with corticosteroid injection, physically therapies, saline solution injection and control groups. The use of HA in patients with rotator cuff tears improve
VAS and functional score in all trials that we have analyzed.

Conclusion: intra-articular injection with HA is effective in reducing pain and improving function in shoulder with rotator cuff tears and without severe adverse reaction.

Level of evidence: Level I.

KEY WORDS: cuff tears, glenohumeral, hyaluronic acid, hyaluronate, shoulder, viscosupplementation.

\section{Introduction}

Rotator cuff pathology is the main cause of shoulder pain and disability. The causes arises from a multivariate etiology, often due to age-related chronic degeneration in which play a role the decrease in collagen synthesis, increase in free radicals expression and metabolism imbalance in favor of catabolic activity ${ }^{1}$. Classically, the diagnosis is clinical, characterized by pain, mostly during the night, severe disability and functional impairment $^{2,3}$. MRI and US may usefully this diagno$\operatorname{sis}^{4,5}$. The first management is conservative, including oral anti-inflammatory drugs, topic agents, cortisone injections, physical therapies, and joint rehabilitation exercises $^{6-8}$. In the past, much has been published on the use of steroid and local anesthetic injections, administered alone or in combination to other therapies. On the other hand, many evidences have corroborated their potential effects as negative on the collagen matrix of tendons and ligaments ${ }^{9-14}$. In the substance, compared to other treatments, effective in the mid and long term, the benefits after these injections could be valid only in the short-term ${ }^{15}$.

Clinical trials have confirmed that HA may be effective for management of tendons disorders. Specifically, in Achilles tendinopathy, the tendon healing process is improved and the formation of adhesions in reduces by the regulation of the expression of vascular endothelial growth factor (VEGF) and type IV collagen ${ }^{16}$. After hand surgery, it improves the motion of fingers lubricating the tendon surface and reducing friction and adhesions ${ }^{17,18}$.

Few papers have investigated the effects of HA in rotator cuff disorders, and no systematic review have been performed on this matter. The aim of the present review is to summarize all papers published inherently to the injection of HA for management of cuff tendinopathy, in terms of feasibility, safety, and efficacy. 


\section{Material and methods}

\section{Criteria for consideration}

We included randomized clinical trials, prospective and retrospective studies reporting on clinical and functional outcomes in patients who had undergone sub-acromial or intra-articular injections of HA for management of rotator cuff pathology. Given the linguistic capabilities of the research team, we considered only papers published in English language. We performed a broad search for relevant studies published up to August 2014 in Medline (http://www.ncbi.nlm.nih.gov/sites/entrez/); Ovid (http://www.ovid.com); Cochrane Reviews (http://www.cochrane.org/reviews/), Google Scholar, Enbase database. Combined key words for the search were "cuff tears", "glenohumeral", "hyaluronic acid", "hyaluronate", "shoulder", "viscosupplementation", with no limit for year of publication. We identified 178 publications. Two Authors (MB and ADB) reviewed the abstract of each publication, and selected or excluded the study according to the text of the abstract. The article was excluded if the abstract was not available. In addition, screening the reference lists of relevant studies, articles not identified at the first electronic search were included. All journals were considered, and all relevant articles were retrieved. Papers referring to a specific association between cuff disorders and obvious osteoarthritis of the shoulder were excluded.

Biomechanical reports, studies on animals, cadavers, in vitro or animal studies, case reports, literature reviews, technical notes, letters to editors and instructional course were excluded. To qualify, an article would have to have been published in peer-reviewed journals. We obtained full-text versions of the study if the abstract did not allow to include or exclude it. All search steps, inclusion and exclusion criteria are reported in Figure 1. 178 articles were identified and selected. 32 full-text selected articles were reviewed and discussed by all the Authors; a fully trained orthopedic surgeon with special interest in shoulder surgery and sports disorders (LO) took the final deci- sion, in dubious cases. After further selections, 11 publications relevant to the topic were included.

\section{Outcome measures}

Data were extracted from each study without contacting the Author(s) to verify the accuracy of the data or obtain further information. The visual analogue scale (VAS) assessment was considered as major criterion for clinical success. Data on range of motion (ROM) ${ }^{19-24}$, Constant score ${ }^{25-28}$, shoulder function assessment scale (SFA) and shoulder disability questionnaire (SDQ ${ }^{23}$, short form-12(SF12) ${ }^{24}$, University of California at Los Angeles score (UCLA) ${ }^{20}$, Oxford Shoulder Score and Patient Global Assessment ${ }^{29}$, Shoulder Rating Questionnaires ${ }^{27}$, and activities of daily living (ADL) ${ }^{28}$ were extracted for assessment of clinical and functional outcomes. Rates of complications were also extracted to assess the safety, effectiveness, and reliability of these procedures.

\section{Results}

Eleven studies have been published from 1995 to 2013, all reporting outcomes of patients who had undergone injections of HA for management of rotator cuff tears (Tab. 1). The number of patients varied from $22^{27}$ to $602^{24}$, for a total number of 1102 patients. Specifically, 701 patients underwent intra-articular injections of $\mathrm{HA}, 236$ underwent intra-articular injections of saline solution ${ }^{21,24,26}, 53$ underwent intra-articular injections of methylprednisolone acetate ${ }^{20,23}$, and 35 patients underwent other physical therapies. Different types of HA were used, based on the low or medium molecular weight. The commercial name also differed: Hylan G-F 2022, Fermathron ${ }^{\text {TM } 26}$, Hyruan Plus ${ }^{\circledR 23}$, SportVis ${ }^{\text {TM } 29}$, Jointex ${ }^{27}$, Artz ${ }^{25}$ were used in one study each, a not specified high weight $\mathrm{HA}^{19,20,28}$ and Hyalgan 21,24 in 3 and 2 studies, respectively.

The mean age of patients managed was 59.2 years. The numbers of injections varied: 2 in 1 studies $^{29}, 1$ a week for five weeks in 5 studies $^{19-21,24,25}$, one ${ }^{23,28}$ in 2

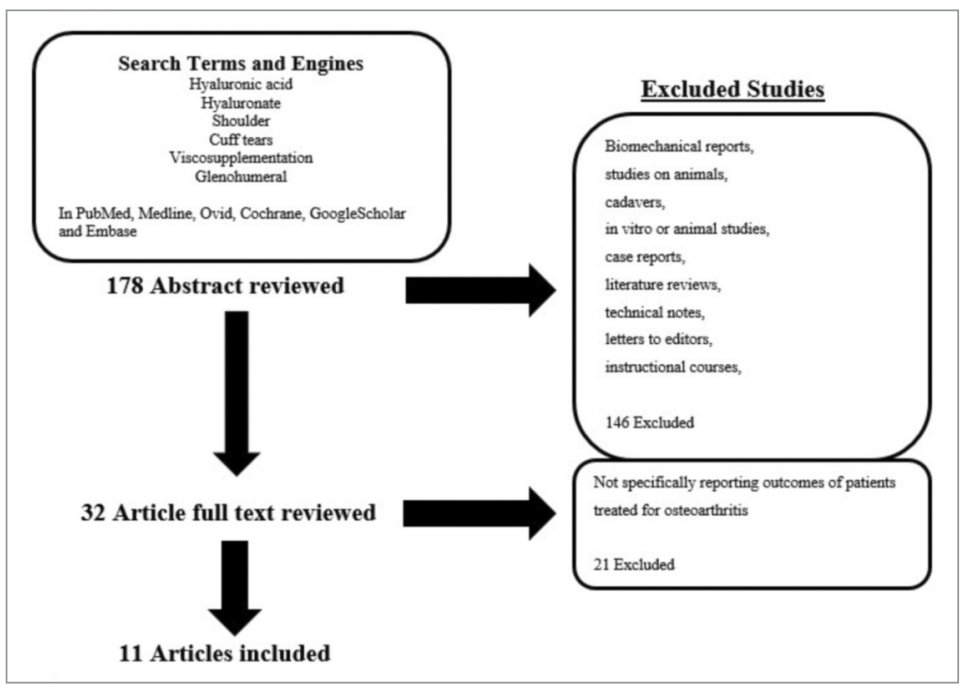

Figure 1. Flow-chart showing the process and results for systematic review and article exclusion. 
Table 1. Studies features.

\begin{tabular}{|c|c|c|c|c|c|c|c|c|}
\hline Bvipst & Inclusion Criteria & Esclusion Criteria & Location & Case & Control & Age & N. patients & Outcomes \\
\hline$h p l b i v-2:: 6-$ & $\begin{array}{l}\text { Subacromial bursitis, tendinitis and } \\
\text { capsulitis. }\end{array}$ & 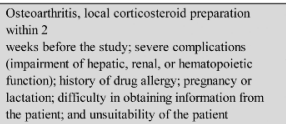 & $\begin{array}{l}\text { glenoid cavity(20) } \\
\text { or the } \\
\text { subacromial } \\
\text { bursal(42). }\end{array}$ & $\begin{array}{l}25 \mathrm{mg} \text { HMW HA in } 1 \% \text { solution, } 1 \\
\text { irjections once weekly for } 5 \text { wecks (If } \\
\text { periarlhritis resolved during this period, } \\
\text { treatment was stopped) }\end{array}$ & & Total-65.4(39-86) & Total-62 & $\begin{array}{l}\text { Pair(at rest, on motion, on } \\
\text { pressure),ROM, ADL, } \\
\text { The gobal severity rating. } \\
\text { Global improvement }\end{array}$ \\
\hline$\pi j c b b b-3112 u ̈$ & $\begin{array}{l}\text { Full-thickness rotatorcuff tcar } \\
\text { diagnosed by arthrography or magnetic } \\
\text { resonance imaging }\end{array}$ & $\begin{array}{l}\text { Undergone intra-articular injection of any drugs, } \\
\text { abnormal hepatic or renal function, pregnant, severe } \\
\text { osteartrititic changes of the affected, shoulder foint } \\
\text { were excluded, symptoms resulting from cervical } \\
\text { lesions. }\end{array}$ & $\begin{array}{l}\text { intra-articular } \\
\text { glenohumeral joint }\end{array}$ & $\begin{array}{l}25 \text { mg sodium hyaluronatc(molecular } \\
\text { weight: } 80 \times 10)+3 \mathrm{~mL} \text { of } 1 \% \text { lidocaine } \\
\text { once weekly for } 5 \text { weeks }\end{array}$ & $\begin{array}{l}2 \mathrm{mg} \text { of dcxannethasone }+3 \mathrm{~mL} \\
\text { of } 1 \% \text { lidocaine once weekly for } \\
5 \text { weeks }\end{array}$ & $\begin{array}{l}\mathrm{Hl}=59.5 \pm 9.1 \\
\mathrm{DI}=62.4+8.6\end{array}$ & $\begin{array}{l}\text { Total }=78 \\
\text { Hl }=38 \text { DI }-40\end{array}$ & UCLA,ROM \\
\hline Nffmoj-3118it- & $\begin{array}{l}\text { Clinical, echographic and magretic } \\
\text { resonance diagnosis of unpraspinatus } \\
\text { tendinosis unresponsive to physical and } \\
\text { medical therapy }\end{array}$ & $\begin{array}{l}\text { Cervical lesions, undergone íntra or periarticular } \\
\text { injection of any drugs }\end{array}$ & $\begin{array}{l}\text { subacrominial } \\
\text { injections under } \\
\text { US guidance }\end{array}$ & $\begin{array}{l}\text { Hyalgann(r), Fidia SpA once a week for } 5 \\
\text { weeks (SH) }\end{array}$ & $\begin{array}{l}\text { sodium chloride solution } \\
\text { injections once a week for } 5 \\
\text { weeks(SC) }\end{array}$ & Total-31-71 & Total-56 & $\begin{array}{l}\text { VAS, ROM, echogaphic } \\
\text { controls }\end{array}$ \\
\hline 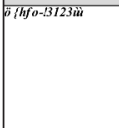 & $\begin{array}{l}\text { Supraspinatus tendinitis through } \\
\text { marifestation and MRI }\end{array}$ & 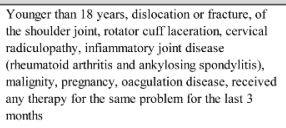 & 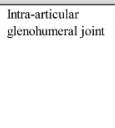 & $\begin{array}{l}2 \mathrm{ml}(16 \mathrm{mg}) \text { of G-F } 20 \text { once a week for } \\
3 \text { weeks }\end{array}$ & $\begin{array}{l}\text { physical therapy agents } \\
\text { (transcutaneous elctrical nerve } \\
\text { stimulation, ultrasound and hot } \\
\text { pack) }\end{array}$ & $\begin{array}{l}\text { HL-58.67 } \\
\text { PTA-52.50 }\end{array}$ & $\begin{array}{l}\text { Total }-24 \\
\text { HI-12 PTA }-12\end{array}$ & $\begin{array}{l}\text { VAS, ROM and functional } \\
\text { evaluation (finction part } \\
\text { of the "Society of the } \\
\text { American Shoulder and } \\
\text { Elbow Surgeons Rating } \\
\text { Scale") }\end{array}$ \\
\hline$C z v 0-3122 z Z$ & $\begin{array}{l}\text { Clinically painful arc and Hawkin's } \\
\text { siegn, positive Neers impingement sign, } \\
\text { partial tear or full thickness tear, } \\
\text { subacromial bursitis among } \\
\text { periarticular soft tissue disorders of the } \\
\text { shoulder in a sonogram }\end{array}$ & $\begin{array}{l}\text { Adhesive capsulitis, history of previous shoulder } \\
\text { surgery, steroid or hyaluronate injection in the } \\
\text { shoulder rof the same causc, hemiplegic shoulder } \\
\text { pain syndrome. }\end{array}$ & $\begin{array}{l}\text { subacromial } \\
\text { injections under } \\
\text { US guidance }\end{array}$ & $\begin{array}{l}40 \mathrm{mg} \text { triamcinolone acetonide }+0.5 \% \\
\text { lidocaine }(5 \mathrm{ml})+\text { hyaluronate Hyruan } \\
\text { Plus } \bar{\Omega}\end{array}$ & $\begin{array}{l}40 \mathrm{mg} \text { triancinolone acetonide } \\
(1 \mathrm{ml}) \text { and } 0.5 \% \text { lidocaine }(5 \mathrm{ml})\end{array}$ & $\begin{array}{l}\mathrm{G} 1-55.5 \pm 12.1 \\
\mathrm{G} 2-55.4 \pm 10.0\end{array}$ & $\begin{array}{l}\text { Total }-26 \\
\mathrm{Gl}=13 \mathrm{G} 2=13\end{array}$ & $\begin{array}{l}\text { VAS, SFA, SDQ, ROM } \\
\text { (Flexion,sbduction,IR,ER) }\end{array}$ \\
\hline Chbjof-B1119ü & $\begin{array}{l}\text { Persistent shoulder pain associated } \\
\text { with limitation of ROM, refractory to } \\
\text { standard treatments. joint ostcoarthritis, } \\
\text { rotator cuff tear (partial or cormplete), } \\
\text { and or primary or secondary adhesive } \\
\text { capsulitis }\end{array}$ & 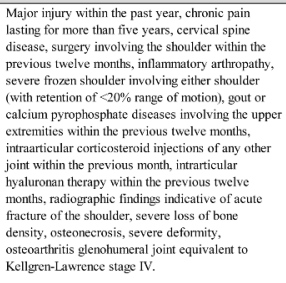 & $\begin{array}{l}\text { intra-articular } \\
\text { glenohumeral joint }\end{array}$ & 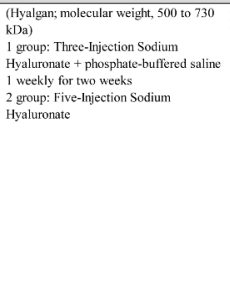 & $\begin{array}{l}\text { phosphate-buffired saline } 1 \\
\text { weekly for five weeks }\end{array}$ & $\begin{array}{l}1 \text { group=-62.3 [SD:12.7] } \\
2 \text { group=63.4 [SD:12.4] } \\
\text { PBS-63.6 [SD:12.3] }\end{array}$ & $\begin{array}{l}\text { Total=602 } \\
1 \text { group }=197 \\
2 \text { group-201 } \\
\text { PBS } 204\end{array}$ & $\begin{array}{l}\text { VAS, ROM, night pain, } \\
\text { Short-Form Health Survey } \\
12 \text { general health } \\
\text { questionaire, use of } \\
\text { rescue medication. }\end{array}$ \\
\hline$D i p-3 / 2 t i t$ & $\begin{array}{l}\text { Pain around the shoulder, positive } \\
\text { impingement sign, positive imaging } \\
\text { diagnosis of rotator cuff pathology } \\
\text { without a complete tear, not respond to } \\
\text { conservative therapy or rehabilitation } \\
\text { for at least } 3 \text { months; aged between } 35 \\
\text { and } 80 \text { years }\end{array}$ & $\begin{array}{l}\text { Rheumatic discases, glenohumeral ssteoarthritis, } \\
\text { full-thickness curf tears, fractures, infections or } \\
\text { tumors, hypersensitivity to hyaluronate }\end{array}$ & $\begin{array}{l}\text { subacromial space } \\
\text { under US } \\
\text { guidance }\end{array}$ & $\begin{array}{l}\text { ARTZ once weckly for consecutive } 5 \\
\text { weeks }\end{array}$ & Placebo & $\begin{array}{l}\text { HI-51.16-7.84 } \\
\text { Placeto }=52.38 \pm 8.95\end{array}$ & $\begin{array}{l}\text { Total-54 } \\
\text { H=25 } \\
\text { Placebo-26 }\end{array}$ & Constant scores,, $\mathrm{VAS}$ \\
\hline Nophi ibefsj-3124ü & $\begin{array}{l}\text { Patients between } 30 \text { and } 80 \text { years old } \\
\text { with shoulder pain, a positive Neer, } \\
\text { Hawkins sign, ultrasonographic } \\
\text { diagnosis of rolator culf paihology } \\
\text { without a complete tear, not respond to } \\
\text { conservativiv treatments or } \\
\text { rehabilitation for at least } 6 \text { months, } \\
\text { signed the informed consent form. }\end{array}$ & 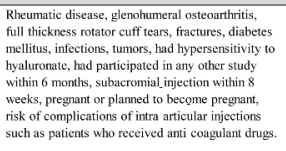 & $\begin{array}{l}\text { subacromial } \\
\text { injections under } \\
\text { US guidance }\end{array}$ & $\begin{array}{l}3 \text { weekly injections of Fernathron }{ }^{\top \mathrm{TA}}, 20 \\
\mathrm{mg} 2 \mathrm{ml} \text { of } \\
\text { sodium hyaluronate. }\end{array}$ & $\begin{array}{l}3 \text { weekly injections of } 0.9 \% \\
\text { normal } \\
\text { saline solution, at } 2 \mathrm{~mL} / \text { syringe. }\end{array}$ & $\begin{array}{l}\text { No sigmificant differences } \\
\text { between two in } \\
\text { demographic data gender } \\
\text { and age }\end{array}$ & $\begin{array}{l}\text { Total:40 } \\
\text { HIl=20 } \\
\text { Control-20 }\end{array}$ & Constant scores, VAS \\
\hline Dppibojop:-311:ü & 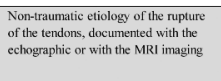 & & $\begin{array}{l}\text { Intra-articular with } \\
\text { anterior access }\end{array}$ & $\begin{array}{l}\text { Jointex starter once a acck for } 3 \text { wecks } \\
\text { followed by a targeted rekabilitiative } \\
\text { intervention (passive } \\
\text { and active assisted kincsittcrapy) }\end{array}$ & & $\mathrm{Agc}-78.1$ & Total-22 & $\begin{array}{l}\text { VAS.Costant Murley, } \\
\text { Shoulder Rating } \\
\text { Questionnaires }\end{array}$ \\
\hline 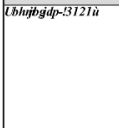 & $\begin{array}{l}\text { Massive rotator curf tear (physical } \\
\text { examination, } x \text {-ray,ultrassound or MRI) } \\
\text { shoulder OA scored as cqual or above } \\
\text { grade II Hamada classification }\end{array}$ & 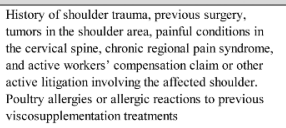 & $\begin{array}{l}\text { Subacrunial } \\
\text { injections under US } \\
\text { guidance }\end{array}$ & $\begin{array}{l}1 \text { injection of high weight sodium } \\
\text { hyaluronate }\end{array}$ & Not treated & 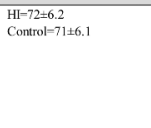 & $\begin{array}{l}\text { Total: } 90 \\
\text { Hl=30 } \\
\text { Control= } 60\end{array}$ & Constant scores,VAS \\
\hline Nfspmb-3124ü & $\begin{array}{l}\text { Persistent shoulder pain fir at least } 4 \\
\text { months, clinical diagnosis of RC } \\
\text { tendinopatly detected with MRI (high } \\
\text { texion signal intensity that was } \\
\text { anatomically intact), no previous } \\
\text { treatment with articular or subacromial } \\
\text { steroid injections within the last } 4 \\
\text { months. }\end{array}$ & 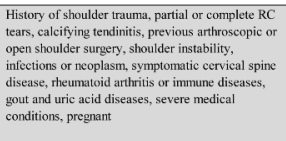 & $\begin{array}{l}\text { subacromial } \\
\text { injections under } \\
\text { US guidance }\end{array}$ & $\begin{array}{l}\text { SportVis }{ }^{\mathrm{MM}} 1 \text { injections once weekly for } \\
2 \text { weeks }\end{array}$ & Physical therapy & $\begin{array}{l}\mathrm{HA}=49+2.35 \\
\mathrm{PT}=51 \pm 2.64\end{array}$ & $\begin{array}{l}\text { Total }-48 \\
\mathrm{H}=25 \text { PT }=23\end{array}$ & $\begin{array}{l}\text { VAS, Constant-Murley } \\
\text { scale, Oxford Shoulder } \\
\text { Scorc, Paticnt Global } \\
\text { Assessment }\end{array}$ \\
\hline
\end{tabular}

studies and $3^{22,24,26,27}$ in 4 studies. Intra-articular injection were undertaken in 5 studies ${ }^{19,20,22,24,27}$ subacromial injections under US guidance were administered in seven studies ${ }^{19,21,23,25,26,28,29}$.

The diagnosis of rotaror cuff tear was mainly on clinical examination, and confirmed at MRI or US scans. The main exclusion criteria were inflammatory arthritis, crystalline synovitis, avascular necrosis, rapidly progressive disease, history of sepsis, trauma, and previous surgery to the shoulder, intra-articular injections of corticosteroids to the shoulder at least 6 months before the treatment, neoplasm, and painful conditions to the cervical spine (Tab. 1).
The follow-up of each study, scores before and after management and adverse reactions are reported in Table 2.

\section{Complications}

In all studies, no serious device-related adverse events were observed. A mild vagal reaction occurred in 1 of 30 patients $(3 \%)^{28}$. In another study 7 patients $(3.2 \%)$ in the five-injection hyaluronate group, 3 patients $(1.4 \%)$ in the three-injection hyaluronate group, and 7 patients $(1.4 \%)$ in the control group complained of persistent pain at the injection site ${ }^{24}$ (Tab. 2). 
Table 2. Clinical results, follows up and complication.

\begin{tabular}{|c|c|c|c|c|}
\hline Authors & Pre-treatment scores & Follow-Up & Post-treatment scores & Complication \\
\hline Ipl $b\{v-12:: 6-$ & $\begin{aligned} \text { ROM= } & \text { Extension } 33.4 \pm 14.7 \\
& \text { Flexion } 118.2 \pm 37.8 \\
& \text { Adduction } 14.6 \pm 16.0 \\
& \text { Abduction } 100.2 \pm 36.8 \\
& \text { Internal rotation } 63.8 \pm 26.8 \\
& \text { External rotation } 39.3 \pm 32.1\end{aligned}$ & $8.16 \pm 0.88 w$ & $\begin{array}{l}\text { Pain at rest- } 39 \text { Improved, } 13 \text { Unchanged, } 0 \text { Aggravated, } 52 \text { Total Assessed, } 75.0 \text { Improvement Rate (\%) } \\
\text { Pain on motion= } 42,15,0,57,73.7 \\
\text { Pain on pressure }=26,7,0,33,78.8 \\
\text { ADL: Face washing } 15,16,0,31,48.4 \\
\text { Hair grooming } 30,16,0,46,65.2 \\
\text { Tying a sash behind the back } 32,16,0,48,66.7 \\
\text { Placing hand on opposite shoulder } 20,12,0.32,62.5 \\
\text { Removing upper garments } 23,13,1,37,62.2 \\
\text { ROM: Extension } 35.7 \pm 14.5<0.01(35 \text { patient) } \\
\text { Flexion } 130.0 \pm 35.8<0.001(55) \\
\text { Adduction } 17.9 \pm 18.8<0.05(35) \\
\text { Abduction } 116.0 \pm 35.6<0.001(58) \\
\text { Internal rotation } 68.9 \pm 22.2<0.01 \text { (37) } \\
\text { External rotation } 47.9+31.9<0.001 \text { ( } 38) \\
\text { Global improvement rating: } 32(51.6 \%) \text { of } 62 \text { patients-moderately improved or better } \\
51(82.3 \%) \text { of } 62 \text { patients-slightly improved or better. }\end{array}$ & none \\
\hline Ti jcblb-13112ì & $\begin{array}{l}\text { Patient background and ROM=NS between two groups } \\
\text { UCLA score(satisfaction patient)= H: } 13.6 \pm 2.6 \\
\text { DI: } 11.9 \pm 3.6 \text { (NS) }\end{array}$ & $4,24 \mathrm{w}$ & $\begin{array}{l}\text { UCLA score(satisfaction patient=16): } \\
\text { HI (16p): } 27.6 \pm 3.1(4 \mathrm{w}), 26.2 \pm 3.1(24 \mathrm{w}) \mathrm{P}<.0001 \\
\text { DI }(15 \mathrm{p}): 26.5 \pm 2.0(4 \mathrm{w}), 25.3 \pm 2.5(24 \mathrm{w}) \mathrm{P}<.0001 \\
\text { ROM in abduction (24w): significantly different }\end{array}$ & none \\
\hline Nfipoj-13118it & $\begin{array}{r}\mathrm{VAS}=\mathrm{SH}=8.7 \\
\mathrm{SC}=8.5\end{array}$ & $\begin{array}{l}4 \mathrm{w}, 3 \mathrm{SH}+\mathrm{SC} \\
6,12 \mathrm{~m} \mathrm{SH} \text { only }\end{array}$ & $\begin{array}{l}\text { VAS=SH=2.8 }(4 \mathrm{w}), 3.1(12 \mathrm{w}), 3.8(6 \mathrm{~m}), 5.1(1 \mathrm{y}) \\
\quad \mathrm{SC}=8.0(4 \mathrm{w}), 8.1(12 \mathrm{w}), \\
\text { ROM=unchanged } \\
\text { echographic controls= no no substantial differences in aspects regarding betwen two groups }\end{array}$ & none \\
\hline$\ddot{\partial}\{h f o-l 3123 \ddot{u} u$ & 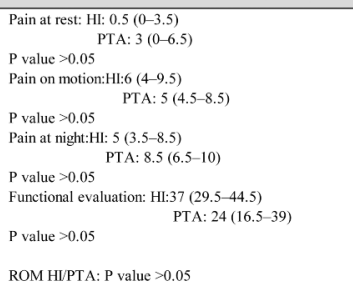 & $3 \mathrm{w}, 3 \mathrm{~m}, 4 \mathrm{y}$ & 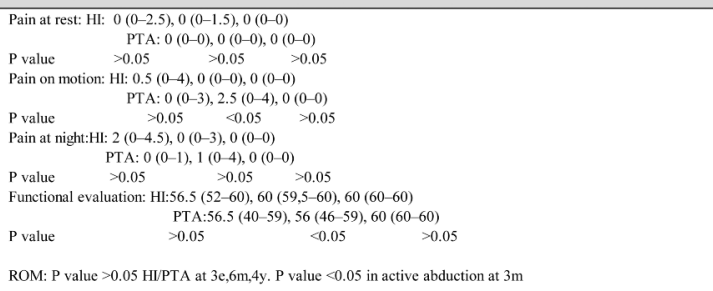 & none \\
\hline$C z v 0-13122 \ddot{Z}$ & 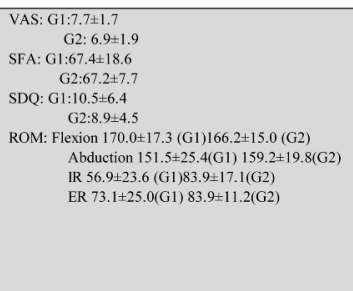 & $\begin{array}{l}\text { Iw after } 1 \text { inj, Iw } \\
\text { after 2inj, 2w after } \\
3 \text { inj }\end{array}$ & 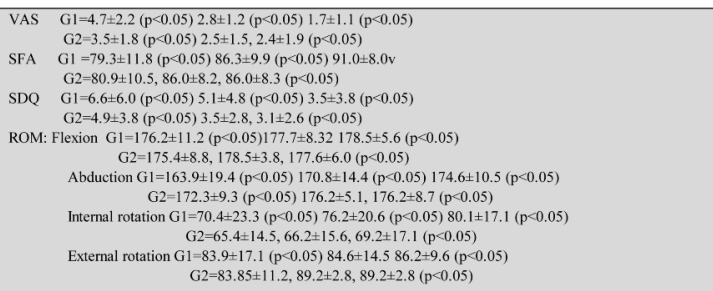 & none \\
\hline Crhjof - -3119й & & $7,9,13,17,26 \mathrm{w}$ & 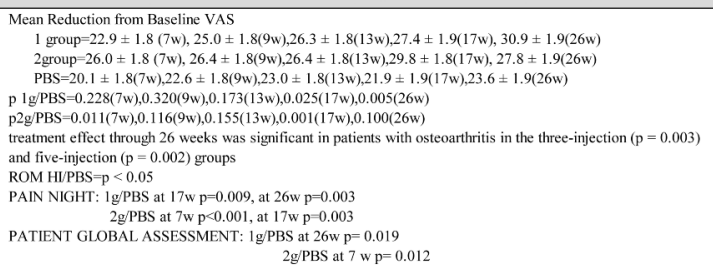 & $\begin{array}{c}\text { The most } \\
\text { frequently averse } \\
\text { event: injection- } \\
\text { site pain }(1 \mathrm{~g}: 3,2 \%, \\
2 \mathrm{2g:1,4 \%} \\
\text { PBS: } 1,4 \%)\end{array}$ \\
\hline Di pv--B121ù & $\begin{array}{l}\text { Constant score: } \begin{aligned} \mathrm{HI}=61.64 \pm 13.37 \\
\text { Placebo }=64.89 \pm 9.46\end{aligned} \\
\text { VAS: } \mathrm{Hl}-6.36 \pm 1.35 \\
\text { Placebo }=6.46 \pm 1.27\end{array}$ & $\begin{array}{l}1 \mathrm{w}, 6 \mathrm{w}, 2 \mathrm{y} \text { after } \\
5 \text { injections }\end{array}$ & $\begin{array}{l}\text { Constant score: } \mathrm{HI}=72.48+16.46(1 \mathrm{w}), 79.24+13.09(6 \mathrm{w}) \\
\text { Placebo }-72.42+11.75(1 \mathrm{w}), 69.07+13.29(6 \mathrm{w}) \\
\text { VAS: } \mathrm{Hl}=4.20 \pm 1.76(1 \mathrm{w}), 3.04+2.03(6 \mathrm{w}) \\
\text { Placebo }=4.77 \pm 1.75(1 \mathrm{w}), 5.12+2.42(6 \mathrm{w})\end{array}$ & none \\
\hline Nphi ibefsj-13124ì & $\begin{aligned} \text { Constant score: } \mathrm{HI}=37.7 \pm 9.9 \\
\text { Control }=40.5 \pm 11.3\end{aligned}$ & $1,2,3,12 \mathrm{w}$ & $\begin{array}{l}\text { Constant score: } \mathrm{HI}=70.4 \pm 16.9(12 \mathrm{w}) \\
\quad \text { Control }=50.7 \pm 13.2(12 \mathrm{w}) \quad \mathrm{P}=0.00\end{array}$ & none \\
\hline & $\begin{array}{l}\text { VAS: } \mathrm{Hl}=9.35 \pm 1.1 \\
\text { Control }=9.5 \pm 0.8\end{array}$ & & 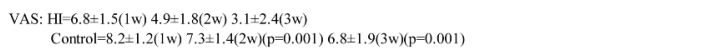 & \\
\hline 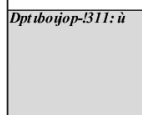 & & $1,3,6 \mathrm{~m}$ & $\begin{array}{l}\text { Friedman test V.A.S. } \mathrm{p}<0.0005 \\
\text { Costant Murley } \mathrm{p}<0.0005 \\
\text { ShoulderRating Quostionnaire } \mathrm{p}<0.0005 \\
\text { Flexion } \mathrm{p}<0.0005 \\
\text { Abduction } \mathrm{p}<0.0005\end{array}$ & none \\
\hline Wbhiphgdp--3121ü & 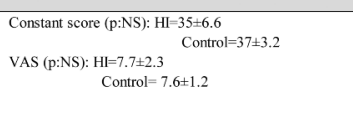 & $1,2,3,4,5,6 \mathrm{~m}$ & 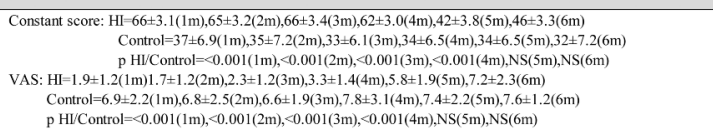 & $\begin{array}{c}\text { mild vagal reaction } \\
\text { in 1/30 patients } \\
(3 \%)\end{array}$ \\
\hline Nfsphb-l3124ù & $\begin{array}{l}\text { VAS HI }=7.48+1.61 \\
\text { PT }=7.17 \pm 1.23(\mathrm{p}: 0.9671) \\
\text { CS }=\text { HI }=53.08 \pm 1.04 \\
\text { PT }=52.91 \pm 2.10(\mathrm{p}: 0.9539) \\
\text { OSS }=\text { HI }=23.28 \pm 0.98 \\
\text { PT }=26.21 \pm 1.08(\mathrm{p}: 0.7841)\end{array}$ & $2,4,12,24 w$ & 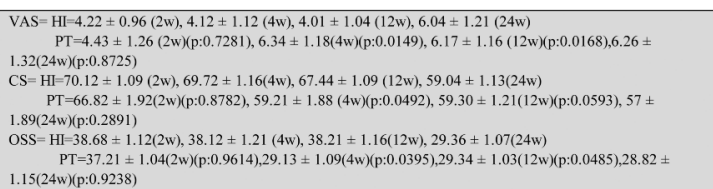 & none \\
\hline
\end{tabular}

\section{Discussion}

The main finding of the present study is that HA injec- tions improve symptoms and function in patients with rotator cuff disorders, without side effects and reactions. On the hand, oral NSAIDs administrations and 
cortisone injections, if prolonged, may be contra-indicated in elderly patients with comorbidities ${ }^{30-32}$ such as diabetes or hypertension. In fact, the HA is physiologically present in the synovial fluid ${ }^{33}$ : its main role is to lubrificate the joint, it exerts mechanical and biological functions, in terms of anti-adhesive shock absorber and articular stabilizer against shear stresses $^{34}$, and it presents some analgesic effects ${ }^{35}$.

The HA is a polymer of disaccharides, composed by Dglucuronic acid and D-N-acetyl-glucosamine, synthesized by a class of integral membrane proteins. It is present in the extracellular matrix, and is a biomechanical and functional element of the articular cartilage ${ }^{36}$.

Specifically, the HA is viscoelastic, lubrificates and protects the articular surface and cartilage from stress and friction forces ${ }^{37}$.

In vitro, HA reduces the gliding resistance after flexor tendon repair ${ }^{38}$. It has an anti-inflammatory role, mediated by the regulation of the concentration of prostaglandin $\mathrm{E} 2, \mathrm{C} 4 \mathrm{~S}$ and interleukin-1 within the synovial fluid, the migration of leukocyte, leukocyte phagocytosis, lymphocyte proliferation, improving significantly pain and discomfort ${ }^{39,40}$. It reduces the concentration of interleukin-6, stimulates synovial fibroblasts to produce endogenous $\mathrm{HA}$, and regulates the expression of endothelial growth factor and type IV collagen ${ }^{16}$ (pro-angiogenic effect) ${ }^{41}$. In one in vivo study on sheep models, HA reduces synovial hyperplasia, inflammation, fibrosis and neovascularization after meniscectomy ${ }^{42}$, limiting the degeneration of cartilages. It was supposed to increase angiogenesis after removal of the anterior cruciate ligament in Wistar rats 43,44 , and to decrease toll-like receptor 4 (TLR-4) and the TLR-2 expression (receptors that play an important role in the arthritis mechanism) in mouse 45 . This systematic review evaluates the effects of HA injections in patients with rotator cuff tears. Different scores and scales were used in the studies. The VAS score improved in all studies examinated in this review. In two studies ${ }^{20,23}$ comparing HA vs methylprednisolone acetate injections, better clinical results and symptoms were recorded after administration of $\mathrm{HA}$. In particular, Byun ${ }^{23}$ showed better results in terms of ROM, proving that HA may improve motion and function of the shoulder, especially in active internal rotation.

Four studies ${ }^{21,24-26}$ comparing patients undergoing HA vs phosphate-buffered saline injections reported good clinical results and pain relief. In particular, Blaine ${ }^{24}$ showed better ROM recovery, reduced pain at night, and significantly higher overall satisfaction in the HA group; Meloni ${ }^{21}$ showed a significant difference in the improvement of clinical symptoms and recovery of functional status in patients at 1 month after the end of the HA infiltrative cycle, in particular HA group VAS score was 2.8 respect 8.0 in the sodium chloride solution group.

$\mathrm{Chou}^{25}$ and Moghtaderi showed a significantly improvement in Constant score and VAS scores at 12 weeks and 6 weeks, respectively.

Two studies ${ }^{22,29}$ evaluated the use of HA compared to the use of physical therapies, concluding that $\mathrm{HA}$ injections are safe and effective for patients with rotator cuff pathology.
There are several limitations to the present investigation. Specifically, few studies evaluated the effects of $\mathrm{HA}$; and different clinical scores were used making it difficult to compare the results.

\section{Conclusion}

Intra-articular injections of HA are effective to reduce pain and improve the function of the shoulder in patients with rotator cuff pathology, with no severe complications or adverse reactions. It could be used as an alternative to cortisone or other oral drugs, exploiting its biomechanical and biochemical properties. Further randomized controlled studies are needed to better understand which is the most effective molecular weight of HA, how often and in which grade of lesions it should be injected.

\section{Conflict of interest}

We declare none conflict of interest.

The Authors declare that this mini-review was conduct according ethically to international standards and as required by the journal as described ${ }^{46}$.

\section{References}

1. Frizziero A, Vittadini F, Gasparre G, Masiero S. Impact of oestrogen deficiency and aging on tendon: concise review. Muscles Ligaments Tendons J. 2014;4(3):324-328.

2. Herrmann SJ, Izadpanah K, Südkamp NP, Strohm PC. Tears of the Rotator Cuff. Causes - Diagnosis - Treatment. Acta Chir Orthop Traumatol Cech. 2014;81(4):256-266.

3. Giai Via A, De Cupis M, Spoliti M, Oliva F. Clinical and biological aspects of rotator cuff tears. Muscles Ligaments Tendons J. 2013;3(2):70-79.

4. Lenza M, Buchbinder R, Takwoingi Y, Johnston RV, Hanchard NC, Faloppa F. Magnetic resonance imaging, magnetic resonance arthrography and ultrasonography for assessing rotator cuff tears in people with shoulder pain for whom surgery is being considered. Cochrane Database Syst Rev. 2013;9:CD009020.

5. Jain NB, Collins J, Newman JS, Katz JN, Losina E, Higgins LD. Reliability of Magnetic Resonance Imaging Assessment of Rotator Cuff: The ROW Study. PM R. 2014.

6. Burbank KM, Stevenson JH, Czarnecki GR, Dorfman J. Chronic shoulder pain: Part II. Treatment. Am Fam Physician. 2008;77:493-497.

7. Van Baar ME, Assendelft WJ, Dekker J, Oostendorp RA, Bijlsma JW. Effectiveness of exercise therapy in patients with osteoarthritis of the hip or knee: A systematic review of randomized clinical trials. Arthritis Rheum. 1999;42:1361-1369.

8. Smidt N, de Vet HC, Bouter LM, et al. Effectiveness of exercise therapy: a best-evidence summary of systematic reviews. Aust J Physiother. 2005;51:71-85.

9. Mikolyzk DK, Wei AS, Tonino P, et al. Effect of corticosteroids on the mechanical strength of rat rotator cuff tendon. J Bone Joint Surg Am. 2009;91:1172-1180.

10. Stannard JP, Bucknell AL. Rupture of the triceps tendon associated with steroid injections. Am J Sports Med. 1993;21:482-485.

11. Tillander B, Franzen LE, Karlsson MH, Norlin R. Effect of steroid injections on the rotator cuff: an experimental study in rats. J Shoulder Elbow Surg. 1999;8:271-274. 
12. Van der Windt DA, Bouter LM. Physiotherapy or corticosteroid injection for shoulder pain? Ann Rheum Dis. 2004;62:385-387.

13. Akpinar S, Hersekli MA, Demirors H, Tandogan RN, Kayaselcuk $F$. Effects of methylprednisolone and betamethasone injections on the rotator cuff: an experimental study in rats. Adv Ther. 2002;19(4):194-201.

14. Oliva F, Osti L, Padulo J, Maffulli N. Epidemiology of the rotator cuff tears: a new incidence related to thyroid disease. Muscles Ligaments Tendons J. 2014;4(3):309-314.

15. Coombes BK, Bisset L, Vicenzino B. Efficacy and safety of corticosteroid injections and other injections for management of tendinopathy: a systematic review of randomised controlled trials. Lancet. 2010;376(9754):1751-1767.

16. Halici M, Karaoglu S, Canoz O, Kabak S, Baktir A. Sodium hyaluronate regulating angiogenesis during Achilles tendon healing. Knee Surg Sports Traumatol Arthrosc. 2004; 12(6):562-567.

17. Riccio M, Battiston B, Pajardi G, et al.; Study Group on Tendon Adhesion of Italian Society of Hand Surgery. Efficiency of Hyaloglide in the prevention of the recurrence of adhesions after tenolysis of flexor tendons in zone II: a randomized, controlled, multicentre clinical trial. J Hand Surg Eur Vol. 2010;35(2):130-138.

18. Wu YF, Tang JB. Tendon healing, edema, and resistance to flexor tendon gliding: clinical implications. Hand Clin. 2013; 29(2):167-178

19. Itokazu M, Matsunaga T. Clinical evaluation of high-molecularweight sodium hyaluronate for the treatment of patients with periarthritis of the shoulder. Clin Ther. 1995;17(5):946-955.

20. Shibata Y, Midorikawa K, Emoto G, Naito M. Clinical evaluation of sodium hyaluronate for the treatment of patients with rotator cuff tear. J Shoulder Elbow Surg. 2001;10(3):209-216.

21. Meloni F, Milia F, Cavazzuti M, Doria C, Lisai P, Profili S, Meloni GB. Clinical evaluation of sodium hyaluronate in the treatment of patients with sopraspinatus tendinosis under echographic guide: experimental study of periarticular injections. Eur J Radiol. 2008;68(1):170-173.

22. Ozgen M, Fırat S, Sarsan A, Topuz O, Ardıç F, Baydemir C. Short- and long-term results of clinical effectiveness of sodium hyaluronate injection in supraspinatus tendinitis. Rheumatol Int. 2012;32(1):137-144

23. Byun SD, Park DH, Choi WD, Lee ZI. Subacromial Bursa Injection of Hyaluronate with Steroid in Patients with Peri-articular Shoulder Disorders. Ann Rehabil Med. 2011;35(5):664-672.

24. Blaine T, Moskowitz R, Udell J, et al. Treatment of persistent shoulder pain with sodium hyaluronate: a randomized, controlled trial. A multicenter study. J Bone Joint Surg Am. 2008;90(5):970-979.

25. Chou WY, Ko JY, Wang FS, Huang CC, Wong T, Wang CJ, Chang HE. Effect of sodium hyaluronate treatment on rotator cuff lesions without complete tears: a randomized, doubleblind, placebo-controlled study. J Shoulder Elbow Surg. 2010;19(4):557-563.

26. Moghtaderi A, Sajadiyeh S, Khosrawi S, Dehghan F, Bateni V. Effect of subacromial sodium hyaluronate injection on rotator cuff disease: A double-blind placebo-controlled clinical trial. Adv Biomed Res. 2013;2:89.

27. Costantino $\mathrm{C}$, Olvirri $\mathrm{S}$. Rehabilitative and infiltrative treatment with hyaluronic acid in elderly patients with rotator cuff tears. Acta Biomed. 2009;80(3):225-229.

28. Tagliafico A, Serafini G, Sconfienza LM, Lacelli F, Perrone N, Succio G, Martinoli C. Ultrasound-guided viscosupplementation of subacromial space in elderly patients with cuff tear arthropathy using a high weight hyaluronic acid: prospective open-label non-randomized trial. Eur Radiol. 2011;21(1):182-187.

29. Merolla G, Sperling JW, Paladini P, Porcellini G. Efficacy of Hylan $\mathrm{G}-\mathrm{F} 20$ versus 6-methylprednisolone acetate in painful shoulder osteoarthritis: a retrospective controlled trial. Musculoskelet Surg. 2011;95(3):215-224.
30. Nelson AE, Allen KD, Golightly YM, Goode AP, Jordan JM. A systematic review of recommendations and guidelines for the management of osteoarthritis: The Chronic Osteoarthritis Management Initiative of the U.S. Bone and Joint Initiative. Semin Arthritis Rheum. 2014;43(6):701-712.

31. Frizziero A, Maffulli N, Masiero S, Frizziero L. Six-months pain relief and functional recovery after intra-articular injections with hyaluronic acid ( $\mathrm{mw} 500-730 \mathrm{kDa}$ ) in trapeziometacarpal osteoarthritis. Muscles, Ligaments and Tendons J. 2014; 4(2):256-261.

32. Foti C, Cisari C, Carda S, Giordan N, Rocco A, Frizziero A, Della Bella G. A prospective observational study of the clinical efficacy and safety of intra-articular sodium hyaluronate in synovial joints with osteoarthritis. Eur J Phys Rehabil Med. 2011;47(3):407-415.

33. Ghosh P, Guidolin D. Potential mechanism of action of intraarticular hyaluronan therapy in osteoarthritis: are the effects molecular weight dependent? Semin Arthritis Rheum. 2002; 32(1):10-37.

34. Cullis-Hill D, Ghosh P. The role of hyaluronic acid in joint stability - a hypothesis for hip displasia and allied disorders. Med Hypotheses 1987;23:171-185.

35. Saito S, Furuya T, Kotake S. Therapeutic effects of hyaluronate injections in patients with chronic painful shoulder: a meta-analysis of randomized controlled trials. Arthritis Care Res (Hoboken). 2010;62(7):1009-1018.

36. Salamanna F, Frizziero A, Pagani S, et al. Metabolic and cytoprotective effects of in vivo peri-patellar hyaluronic acid injections in cultured tenocytes. Connect Tissue Res. 2015; 56(1):35-43.

37. Frizziero A, Salamanna F, Giavaresi G, et al. Hyaluronic acid injections protect patellar tendon from detraining-associated damage. Histol Histopathol. 2015:11605.

38. Kolodzinskyi MN, Zhao C, Sun YL, An KN, Thoreson AR, Amadio PC, Moran SL. The effects of hylan g-f 20 surface modification on gliding of extrasynovial canine tendon grafts in vitro. J Hand Surg Am. 2013;38(2):231-236.

39. Goto M, Hanyu T, Yoshio T, et al. Intra-articular injection of hyaluronate (SI-6601D) improves joint pain and synovial fluid prostaglandin E2 levels in rheumatoid arthritis: a multicenter clinical trial. Clin Exp Rheumatol. 2001;19:377-383.

40. Yasui T, Akatsuka M, Tobetto K, Hayaishi M, Ando T. The effect of hyaluronan on interleukin-1 alpha-induced prostaglandin E2 production in human osteoarthritic synovial cells. Agents Actions. 1992;37(1-2):155-156.

41. Henrotin Y, Lambert C, Richette P. Importance of synovitis in osteoarthritis: evidence for the use of glycosaminoglycans against synovial inflammation. Semin Arthritis Rheum. 2014; 43(5):579-587.

42. Cake MA, Smith MM, Young AA, Smith SM, Ghosh P. Read Synovial pathology in an ovine model of osteoarthritis: effect of intraarticular hyaluronan (Hyalgan). Clin Exp Rheumatol. 2008;26(4):561-567.

43. Tsai WY, Wu JL, Liu CC, Cherng CH, Tsai RY, Jean YH, Wong CS. Early intraarticular injection of hyaluronic acid attenuates osteoarthritis progression in anterior cruciate ligament-transected rats. Connect Tissue Res. 2013;54(1):49-54.

44. Wiig ME, Amiel D, VandeBerg J, Kitabayashi L, Harwood FL, Arfors KE. The early effect of high molecular weight hyaluronan (hyaluronic acid) on anterior cruciate ligament healing: an experimental study in rabbits. J Orthop Res. 1990;8(3):425-434.

45. Campo GM, Avenoso A, Nastasi G, et al. Hyaluronan reduces inflammation in experimental arthritis by modulating TLR-2 and TLR-4 cartilage expression. Biochim Biophys Acta. 2011;1812(9):1170-1181.

46. Padulo J, Oliva F, Frizziero A, Maffulli N. Muscles, Ligaments and Tendons Journal. Basic principles and recommendations in clinical and field science research. MLTJ. 2013;4:250-252. 